\title{
Zwischen Fiktion, Dokufiktion und Metafiktion: Umberto Ecos Roman Il cimitero di Praga im Kontext seiner Recherchen zu den Weisen von Zion
}

\section{1 Über Ecos Romane im Kontext des Rahmenthemas Fakten und Fiktionen}

Ecos Romane, die als Darstellungen erfundener Ereignisse einerseits klar dem Bereich literarischer Fiktionen zuzuordnen sind, nehmen andererseits nicht allein Bezug auf vielfältige Wissensbestände, die im Kontext der „Enzyklopädie“ unserer Kultur als historisches „Fakten“-Wissen gelten (inklusive Faktenwissen über literarische und andere Fiktionen); ${ }^{1}$ diese Romane sind außerdem inhaltlich-thematisch vielfältig mit Ecos nicht-fiktionalen Texten vernetzt, erörtern analoge Ge-

1 „Enzyklopädien“ im Sinne Ecos sind (gedachte) Großkomplexe von Vorstellungsinhalten - und in dieser Eigenschaft nicht unbedingt dasselbe wie „Lexika“, „Wörterbücher“ und andere Kompendien des Wissens, die sich medial konkretisieren. Vgl. Mersch, Dieter. Umberto Eco. Zur Einführung. Hamburg: Junius 1993, 220. Wie Mersch erklärt, entlehnt Eco den Begriff „Enzyklopädie“ aus Wilson, N. L. „Linguistic Butter and Philosophical Parships“. In: Journal of Philosophy 64 (1967), 55-67. Wilson wende sich, so Mersch, gegen die Gleichsetzung einer „Enzyklopädie“ mit einem Wörterbuch mit dem Argument, „daß in die Bedeutungen historisch übermittelte Vorstellungen eingehen, die nicht mittels stereotyper Eintragungen geklärt werden können.“ (Mersch: Umberto Eco, 220.) Bei Eco werde „der Begriff der Enzyklopädie auf die gesamte Geschichte des Wissens ausgeweitet - und so zur Grundlagenkategorie seiner gesamten semiotischen Bedeutungstheorie.“ (Mersch: Umberto Eco, 220.) Maßgebliche Ausführungen Ecos zum Konzept der semiotischen Enzyklopädie finden sich in den Schriften: Semiotik. Entwurf einer Theorie der Zeichen. Aus dem Italienischen von Günter Memmert. München: Fink 1987 [1976], 143-146, 162-166; Lector in fabula. Die Mitarbeit der Interpretation in erzählenden Texten. Aus dem Italienischen von Heinz-Georg Held. München: Carl Hanser 1987 [1977], 15-16, 94-106; Semiotik und Philosophie der Sprache. Aus dem Italienischen von Christiane Trabant-Rommel und Jürgen Trabant. München: Fink 1985 [1984], 107-132. - Das, was Eco als die „regulative Hypothese“ eines „umfassenden semantischen Universums“ bezeichnet (Eco: Semiotik, 179), ist eine ideale Konstruktion, ähnlich dem mittelalterlichen Konzept des ,speculum mundi‘. Es umfasst, so Merschs Paraphrase, „den gesammelten Vorrat an Weisheiten und wissenschaftlichen Irrtümern wie an Vorurteilen, Dogmen, volkstümlichen Legenden, Fiktionen, utopischen Träumereien und Allerweltsbetrachtungen.“ (Mersch: Umberto Eco, 111.) 
genstände, bespiegeln dieselben epistemischen und semiologischen Fragen und wenden sich teilweise denselben historischen Problemen zu. Besonders deutlich wird dies an Ecos Texten über Verschwörungstheorien, über deren Implikationen und Funktionsmechanismen sowie über historische Beispiele - also über ein Themenfeld, das dem Themenfeld um Fakten und Fiktionen per se eng affin ist. ${ }^{2}$

Ecos Werk steht zu weiten Teilen im Zeichen der Reflexion über die Geschichte des Wissens, über auf solch historischem Wissen basierende Beurteilungen von Informationen oder Darstellungen als faktual oder als fiktional sowie über die Transgressionen und Wechselwirkungen zwischen dem als ,faktisch Interpretierten und dem als ,fiktional' Geltenden. Auch und gerade seine Romane bilden im Zeichen dieses Themengeflechts ein thematisches und motivisches Netzwerk. Interpretationsprozesse und ihre Gegenstände, Erfindungen zwischen Täuschung und ästhetischer Fiktion sowie in mehreren Werken auch Verschwörungstheorien stehen hier immer wieder im Mittelpunkt. ${ }^{3}$ Die jeweils konstruierten fiktionalen Geschichten nutzen als Basis dabei reale historische Quellen und die um diese bestehenden Zusammenhänge. Il nome della rosa (dt. Der Name der Rose, 1980) enthält vielfältige Reflexionen über Interpretationen und Fiktionen, und die Fabel illustriert die Rückwirkung von Interpretationen auf die interpretierte Realität. Hier nimmt das prophetische Szenario der Apokalypse die Rolle der Verschwörungstheorie ein, und im Sinn einer self-fulfilling prophecy kommt es zu apokalyptisch erscheinenden Ereignissen. In Il pendolo di Foucault (dt. Das Foucaultsche Pendel, 1988) basteln die drei Hauptfiguren mutwillig an einer an kollektive Phantasmen und Fiktionen anknüpfenden Weltauslegung, die sich wegen ihrer scheinbaren Konsistenz gegen eine rationalere Interpretation der Welt durchsetzt und zur dramatischen Realisierung des Erfundenen führt, ganz entsprechend Ecos theoretischen Modellierungen verschwörungstheoretischer Wirkungszusammenhänge. In Baudolino (2000) erfindet der Protagonist auf der Basis vorliegender Darstellungen, die zu seiner Zeit (dem frühen Mittelalter) als faktuale historiographische und topographische Zeugnisse gelten, ein Reich und seinen Herrscher (den Priesterkönig Johannes), und seine Fiktion macht Realgeschichte (im Rahmen des Romans, dies aber in Anlehnung eben an die reale Geschichte). In La misteriosa fiam-

2 Mit Verschwörungstheorien setzt sich Eco in verschiedenen Schriften auseinander. Neben den Romanen Il cimitero di Praga, Il pendolo di Foucault (dt. Das Foucaultsche Pendel) und Numero zero (dt. Nullnummer) zu nennen sind insbesondere die poetologischen Erörterungen in Im Wald der Fiktionen (Harvard Lectures) sowie Die Fabrikation des Feindes und andere Gelegenheitsschriften (It. Costruire il nemico e altri scritti occasionali).

3 Aus dem Rahmen fällt, was den Rekurs auf Verschwörungstheoretisches angeht, letztlich nur L'isola del giorno prima, wo allerdings ebenfalls über den ontologischen Status von Fiktionen reflektiert wird. 
ma della regina Loana (dt. Die geheimnisvolle Flamme der Königin Loana, 2004) geht es eher nebenher um eine Verschwörungstheorie und ihren Einfluss auf die realen Verhältnisse im Italien der zweiten Hälfte des 20. Jahrhunderts, konkreter: um den Glauben von Anhängern Mussolinis an dessen mögliche Rückkehr, Jahrzehnte nach seinem Tod, und um die politischen Implikationen dieses Glaubens. Und in Numero zero (dt. Nullnummer, 2015) geht es um die lügenhaften Prognosen einer rechtspopulistisch gesteuerten Presse und deren Macht über das, was dann tatsächlich geschieht, also wiederum um die realitätsprägende Macht von Fiktionen; als Akteure im Spiel sind wiederum die Mitglieder einer verschwörerischen Geheimgesellschaft.

Wer sich für die Frage der Differenzierbarkeit zwischen und gleichzeitigen Verflechtung von Faktischem und Fiktionalem interessiert, findet in Verschwörungstheorien einen ergiebigen Gegenstand der Erörterung, insbesondere dann, wenn sie sich auf die Realgeschichte auswirken. ${ }^{4}$ Sie kreisen um Verschwörungen (respektive um ausgedachte Rahmenbedingungen von Verschwörungen), die sich ihre Urheber vorstellen, sei es, dass sie selbst daran glauben, sei es, dass sie die Komplotte bewusst in ihren Vorstellungen konstruieren. Wer an das Konstruierte glaubt, glaubt insofern an Fiktionen. Neben den echten Gläubigen stehen allerdings andere Rezipienten der jeweiligen Verschwörungstheorie: die, die aus ihnen politischen und kommerziellen Profit ziehen. Insofern Verschwörungstheorien oft konkrete politisch-praktische Folgen haben, nehmen sie letztlich eine eigentümliche Zwischenstellung zwischen Fiktionen und Fakten ein, denn zu ihren Folgen gehören Strategien zur Verhinderung des (fiktionalen) Verschwörerziels, Maßnahmen zur Bekämpfung der (vermeintlichen) Verschwörer, Bezichtigungen, Diffamierungen, Verfolgungen. Aus etwas Fiktionalem entwickelt sich Faktisches - vor allem auf der Basis einer Interpretation scheinbarer Dokumente, die als Beweise gelesen werden. Ecos Interesse an Verschwörungstheorien steht im Kontext seiner Reflexionen über Aushandlungsprozesse des „Faktischen“ und des „Fiktionalen“ und bildet zugleich ein wichtiges Scharnier zwischen theoretischen Modellierungen von Interpretationsprozessen und dem Wirken als politischer Aufklärer. Gerade am Beispiel verschwörungstheoretischer Fiktionen und ihrer realen politischen Konsequenzen verdeutlicht Eco die Notwendigkeit eines selbstkritischen und methodisch bewussten Interpretierens vermeintlicher Dokumente, Zeugnisse, Beweise.

4 Wichtige Quellen für Eco sind anlässlich der Beschäftigung mit Verschwörungstheorien und insbesondere mit der Geschichte der Protokolle: Cohn, Norman. Warrant for Genocide: The Myth of the Jewish World Conspiracy and the Protocols of the Elders of Zion. New York: Harper \& Row 1966, und Rollin, Henri. L'apocalypse de notre temps. Les dessous de la propagande allemande d'après des documents inédits. Paris: Gallimard 1939. 


\section{Urteile über „Fakten“ und „Fiktionen“ als enzyklopädiebasierte Interpretationen}

Reflexionen über Fakten und Fiktionen prägen Ecos Romane und stehen im Zentrum einer ganzen Reihe theoretischer Schriften, so etwa seiner Poetikvorlesungen mit dem Titel Im Wald der Fiktionen..$^{5}$ Der Akzent liegt stets, wenn auch in kontextbedingt jeweils modifizierter Form, auf der Komplementarität beider Begriffe. Eco unterscheidet begrifflich das Fiktionale vom Nichtfiktionalen (Faktischen), auch wenn für ihn diese Differenzierung nicht auf einen ontologischen Unterschied verweist, sondern auf differierende Interpretationsmodi von (direkten oder indirekten) Erfahrungsinhalten - jeweils im Rahmen einer semiotischen Enzyklopädie. Die zentrale Frage ist die nach den Kriterien der Unterscheidung. Auch zwischen Ecos Strategien im Umgang mit der Differenzierung zwischen Faktischem und Fiktionalem ließe sich nochmals heuristisch differenzieren. Ein erster Ansatz (a) arbeitet mit dem Konzept des „Fiktionspaktes“, das eine Differenz zwischen Faktischem und Fiktionalem voraussetzt bzw. impliziert. Ein zweiter Ansatz (b) orientiert sich am Konzept der „Enzyklopädie“.

(a) Die Rezeption literarischer (und anderer künstlerischer) Fiktionen als Fiktionen begründet Eco im Rekurs auf S. T. Coleridge mit Fiktionsverträgen, was u. a. die Differenzierung zwischen Fiktionen und absichtsvollen Täuschungen bzw. Lügen möglich macht. ${ }^{6}$ „Fiktionalität“ bzw. „Lügenhaftigkeit“ sind also keine absoluten Zuschreibungen, sondern Resultate von Interpretationen. Vergleicht man im Horizont des von Eco bevorzugten Ansatzes des Welten-Vergleichs allerdings fiktionale und wirkliche respektive faktische Welten, so erweisen sich laut Eco die ersteren vielfach als geschlossener, konsistenter, bezogen auf ihre Merkmale ein-

5 Ein wichtiges Thema ist hier - wie schon in Postille a ,Il nome della rosa (dt. Nachschrift zum ,Namen der Rose`) - die Genese, genauer: die Konstruktion fiktionaler Welten.

6 „Die Grundregel jeder Auseinandersetzung mit einem erzählenden Werk ist, daß der Leser stillschweigend einen Fiktionsvertrag mit dem Autor schließen muß, der das beinhaltet, was Coleridge ,the willing suspension of disbelief', die willentliche Aussetzung der Ungläubigkeit nannte. Der Leser muß wissen, daß das, was ihm erzählt wird, eine ausgedachte Geschichte ist, ohne darum zu meinen, daß der Autor ihm Lügen erzählt. Wie John Searle es ausgedrückt hat [„The Logical Status of Fictional Discourse“. In: New Literary History 6 (1975), 319-332], der Autor tut einfach so, als ob er die Wahrheit sagt, und wir akzeptieren den Fiktionsvertrag und tun so, als wäre das, was der Autor erzählt, wirklich geschehen.“ (Eco, Umberto. Im Wald der Fiktionen. Sechs Streifzüge durch die Literatur. Aus dem Italienischen von Burkhart Kroeber. München: dtv 2004 [1994], 103. (Herv. i. O.)) 
facher beurteilbar als letztere; da sie von Texten konstituiert werden, ist klarer entscheidbar als bei letzteren, was zu ihnen gehört und wie sie beschaffen sind. ${ }^{7}$

(b) Urteile über Faktizität und Fiktionalität sind einem zweiten (und letztlich ergiebigeren) Ansatz zufolge Zuschreibungen auf der Basis begrifflich-epistemischer Konstrukte, deren Bedeutung sich jeweils im Rahmen historisch-kultureller Kontexte profiliert. Als Basis entsprechender Zuschreibungen fungiert die (gedachte) Gesamtheit kulturellen Wissens, die Eco (in Spezifikation dieses Terminus) „Enzyklopädie“ nennt; die „Enzyklopädien“ historischer Kulturen umfassen verifizierbares Wissen ebenso wie allgemein geteilte Annahmen über Zustand und Gesetze der Welt. So kann zur „Enzyklopädie“ einer Kommunikationsgemeinschaft das Wissen darüber gehören, dass es „Einhörner“ gibt, welche Eigenschaften diese haben etc. Diese Eigenschaften und das Aussehen von Einhörnern bleiben auch dann Bestandteile der Enzyklopädie, wenn die Existenz des Einhorns allgemein nicht mehr geglaubt wird. Als Bestandteil der Enzyklopädie kann das Wissen über das als solches ,fiktionale‘ Einhorn sogar dazu dienen, ,Faktenwissen‘ zu vermitteln - respektive das, was gerade als Faktenwissen gilt. Die Differenzierung zwischen faktenbezogenem und fiktionalem Einhorn-Wissen ist im Übrigen relativ, wenn man in Betracht zieht, dass die Beobachtungen von Nashörnern ebenfalls in das Einhorn-Wissen eingeflossen sein mögen. ${ }^{8}$

7 „Über die reale Welt sagen wir, daß die Gesetze der universalen Gravitation diejenigen sind, die Isaac Newton formuliert hat, oder daß es wahr ist, daß Napoleon am 5. Mai 1821 auf Sankt Helena gestorben ist. Und doch werden wir [...] bereit sein, unsere Überzeugungen zu revidieren, sobald die Wissenschaft eine neue Formulierung der großen Gesetze des Kosmos vorlegt oder ein Historiker neue Dokumente findet, die beweisen, daß Napoleon bei einem Fluchtversuch auf einem bonapartistischen Schiff gestorben ist. In der Welt der Bücher dagegen werden Aussagen wie ,Sherlock Holmes war Junggeselle` oder, Rotkäppchen wird vom Wolf verschlungen und dann vom Jäger gerettet‘ oder ,Anna Karenina wirft sich vor einen Zug' in alle Ewigkeit immer wahr bleiben und von niemandem widerlegt werden können. Es gibt Leute, die verneinen, daß Jesus Gottes Sohn war, und solche, die sogar seine historische Existenz bezweifeln, [...] und was immer wir darüber denken, wir behandeln sie alle mit Respekt. Aber niemand wird jemanden mit Respekt behandeln, der behauptet, Hamlet habe Ophelia geheiratet oder Superman sei nicht Clark Kent. Literarische Texte sagen uns nicht bloß ausdrücklich, was wir nie mehr in Zweifel ziehen können, sondern sie bedeuten uns auch im Unterschied zur realen Welt mit souveräner Autorität, was in ihnen als relevant zu gelten hat und was wir nicht zum Ausgangspunkt freier Interpretationen nehmen können.“ (Eco, Umberto. „Über einige Funktionen der Literatur“. In: Die Bücher und das Paradies. München: Carl Hanser 2003, 9-24, hier 13-14.)

$8 \mathrm{Vgl}$. Eco, Umberto. Il nome della rosa. Mailand: Bompiani 1980, 318. ,,,Non è detto che non esista. Forse è diverso da come lo rappresentano questi libri. “ “ [,,Wer sagt denn, daß es [das Einhorn] nicht existiert? Aber vielleicht ist es ganz anders, als es in diesen Büchern dargestellt wird. “" Eco, Umberto. Der Name der Rose. München: Carl Hanser 1982 [1980], 404.] Es folgt eine Anspielung auf Berichte Marco Polos über ein Nashorn. 
Weil „Faktizität“ keine Eigenschaft, sondern Produkt einer Zuschreibung ist, weil das Urteil über die Faktizität von Aussagen, die Echtheit von Dokumenten, die Authentizität von Bekundungen stets von anderen Aussagen, Dokumenten, Bekundungen abhängt, lassen sich aber auch Fälschungen, Lügen, Fakes lancieren. Die scheinbar ihre Faktizität bzw. Echtheit bekundenden Dokumente brauchen dazu bloß ihrerseits fingiert bzw. in Täuschungsabsicht produziert oder herangezogen zu werden. In Die Grenzen der Interpretation diskutiert Eco ausführlich verschiedene Definitionen von „Nachahmung“ und „Fälschung“, einem Begriffspaar, das mit dem von „Fiktion“ und „Lüge“ eng verknüpft ist und auf die Polyvalenz der Idee des „Originalen“ verweist.

Geht man von der Normalsprache aus, so scheint jeder zu wissen, was eine Nachahmung oder eine Fälschung ist. [...] Die Definition von Begriffen wie Nachahmung, Fälschung, Pseudepigraph, Falsifikat, Faksimile, unecht, pseudo, apokryph und anderen ist ziemlich kontrovers. Man darf deshalb vermuten, daß viele der Schwierigkeiten bei der Definition dieser Begriffe mit den Schwierigkeiten zusammenhängen, die auftreten, wenn man den Begriff ,Original' oder ,authentisches Objekt' definieren möchte. ${ }^{9}$

Im Zusammenhang der Unterscheidungsproblematik konstruiert Eco einen fiktiven, selbst mit der Grenze zwischen Erfindung bzw. Fiktion und historischen Referenzen spielenden Beispielfall zur Relativität von Unterscheidungskriterien zwischen echten und falschen Dokumenten, zwischen authentischen Quellen und Falsifikaten. ${ }^{10}$ Sein Fazit: Die geläufige Idee davon, was eine ,Fälschung' sei, setze

9 Eco, Umberto. Die Grenzen der Interpretation. Aus dem Italienischen von Günter Memmert. München: dtv 1999 [1990], 217. Vgl. für die folgende Erörterung von Konzeptionalisierungsformen Eco: Die Grenzen der Interpretation, 217-251.

10 „Unsere Kultur scheint [...] ,befriedigende“ Kriterien zum Beweis der Echtheit und zum Aufdecken falscher Identifizierungen entwickelt zu haben. Alle aufgezählten Kriterien sind aber wohl nur anwendbar, wenn ein Richter ,unvollkommene‘ Fälschungen vor sich hat. Gibt es eine ,perfekte‘ Fälschung (vgl. Goodman 1968), die allen philologischen Kriterien widersteht? Oder gibt es Fälle, in denen keinerlei äußerer Beweis zur Verfügung steht und die inneren sehr fragwürdig sind? Man könnte sich folgendes vorstellen: / 1921 behauptet Picasso, ein Porträt von Honorio Bustos Domeq [Pseudonym von Borges/Bioy Casares] gemalt zu haben. Fernando Pessoa schreibt, er habe das Bild gesehen, und lobt es als das größte Meisterwerk von allen, die Picasso je gemalt hat. Viele Kritiker suchen nach dem Bild, aber Picasso sagt, es sei gestohlen worden. / 1945 erklärt Salvador Dalí, er habe dieses Bild in Perpignan wiederentdeckt. Picasso erkennt es offiziell als sein Originalwerk an. Es wird an das Museum of Modern Art als ,Pablo Picasso, Porträt des Bustos Domeq, 1921“ verkauft. / 1950 schreibt Jorge Luis Borges einen Aufsatz (,El Omega de Pablo`), in dem er feststellt: / 1. Picasso und Pessoa haben gelogen, weil niemand 1921 ein Porträt von Domeq gemalt hat. / 2. Es war in jedem Fall unmöglich, 1921 einen Domeq zu porträtieren, weil diese Figur in den vierziger Jahren von Borges und Bioy Casares erfunden worden ist. / 3. Picasso hat das Bild 1945 gemalt und auf 1921 rückdatiert. / 4. Dalí hat das Bild gestohlen und 
ein ,echtes Original‘ als Vergleichsrelat voraus. Doch „alle Kriterien, mittels derer man feststellen kann, ob etwas die Fälschung eines Originals ist“", seien deckungsgleich mit denen,

die es erlauben, festzustellen, ob das Original echt ist. Also kann das Original nicht als Parameter zum Aufdecken von Fälschungen verwendet werden, es sei denn, man akzeptiert blind, daß das, was als Original präsentiert wird, auch unzweifelhaft das Original ist [... . ${ }^{11}$

Doch das sei für den Philologen nicht akzeptabel. ${ }^{12}$ Die Gegenstände der Philologie (Texte und die Frage ihrer Authentizität) und die der Semiotik (insbesondere die semiotische Enzyklopädie) sind analog. Zwischen „Enzyklopädien“ und den Kriterien dafür, dass einzelne Annahmen, Hypothesen, Theorien und Informationen in die ,enzyklopädischen' Wissensbestände einer kulturellen Gemeinschaft eingehen, besteht ein zirkuläres Verhältnis, das mit dem hermeneutischen Zirkel verglichen werden kann, je nach Akzentuierung sogar einem hermeneutischen Zirkel entspricht: „Enzyklopädien“ umfassen ja auch die anerkannten epistemischen Vorgaben entsprechender Urteilsbildung über Faktizität oder Fiktionalität, die dann im Einzelnen über das bestimmen, was als enzyklopädiefähiges Wissen gilt. Im Umgang mit fiktiven Welten orientieren sich Autoren und Leser an der „Enzyklopädie“ der wirklichen Welt.

Die fiktiven Welten sind Parasiten der wirklichen Welt. Es gibt keine Regel, die vorschreibt, wie viele fiktive Elemente in einem Werk akzeptabel sind, es gibt hier im Gegenteil eine große Flexibilität: Formen wie beispielsweise das Märchen veranlassen uns auf Schritt und Tritt zu Korrekturen unseres Wissens von der wirklichen Welt. Doch alles, was im Text nicht aus-

(perfekt) kopiert. Unmittelbar danach hat er das Original vernichtet. / 5. Offensichtlich hat Picasso 1945 seinen eigenen Frühstil perfekt imitiert, und Dalís Kopie war ununterscheidbar vom Original. Sowohl Picasso wie Dalí haben Farben und Leinwand aus dem Jahr 1921 verwendet. / 6. Folglich ist das in New York ausgestellte Werk die bewußte Fälschung einer bewußten Fälschung durch den Autor einer geschichtlichen Fälschung. / 1986 wird ein unbekannter Text Raymond Queneaus gefunden, der behauptet: / 1. Bustos Domeq hat es tatsächlich gegeben, er hieß aber in Wahrheit Schmidt. Alice Toklas hat ihn 1921 maliziöserweise Braque als Domeq vorgestellt, und Braque porträtierte ihn (gutgläubig) unter diesem Namen, wobei er (in Täuschungsabsicht) Picassos Stil imitierte. / 2. Domeq-Schmidt starb bei der Bombardierung Dresdens, wobei alle seine Personalpapiere verlorengingen. / 3. Dalí hat das Porträt tatsächlich 1945 entdeckt und es kopiert. Später zerstörte er das Original. Eine Woche darauf fertigte Picasso eine Kopie von Dalís Kopie an; später wurde Dalís Kopie zerstört. Das ans MOMA verkaufte Bild ist eine von Picasso gemalte Fälschung, die eine von Dalí gemalte Fälschung imitiert, die ihrerseits eine von Braque gemalte Fälschung imitiert. / 4. Er (Queneau) hat all dies vom Entdecker der Hitler-Tagebücher erfahren.“ (Eco: Die Grenzen der Interpretation, 250-251.)

11 Eco: Die Grenzen der Interpretation, 252.

12 Eco: Die Grenzen der Interpretation, 252. 
drücklich als verschieden von der wirklichen Welt erwähnt oder beschrieben wird, muß als übereinstimmend mit den Gesetzen und Bedingungen der wirklichen Welt verstanden werden. ${ }^{13}$

In gewissem Sinn, so Eco, könne die reale Welt aber ebenso als Teil der fiktionalen betrachtet werden wie umgekehrt die fiktionale als Teil der realen Welt. ${ }^{14}$ So elegant der Rekurs auf die „Enzyklopädie“ als gemeinsamen Bezugshorizont zur Beschreibung der ,wirklichen Welt‘ auch erscheint und so viele Probleme im Umgang mit den Implikationen fiktionaler Weltbeschreibungen er auch löst - ein sicheres Fundament für Erörterungen über die Wahrheit und Falschheit von Aussagen bietet er keineswegs. So kann es etwa durchaus sein, dass die Enzyklopädie einer kulturellen Gemeinschaft in Teil-Enzyklopädien von Gruppen zerfällt, die unvereinbar sind - beispielsweise, wenn es um das Wissen über Monster und Geister, Gott und den Teufel geht.

13 Eco: Im Wald der Fiktionen, 112. Vgl. auch: Eco, Umberto. Kant und das Schnabeltier. München: dtv 2003, 371 (It. Kant e l'ornitorinco. Mailand 1997): „Das interessante Problem ist nicht die Frage, ob die fiktiven Personen in derselben Weise wie die realen existieren: Die Antwort darauf lautet ,nein' [... . . Das interessante Problem ist die Frage, weshalb wir uns auf sie in derselben Weise beziehen können, wie wir uns auf reale Personen beziehen, denn wir verstehen uns ausgezeichnet, sowohl wenn wir sagen, daß Napoleon der Gatte von Josephine, wie wenn wir sagen, daß Odysseus der von Penelope war. Das geht deshalb, weil alle Enzyklopädien darin übereinstimmen, daß sie Josephine die Eigenschaft zuschreiben, in zweiter Ehe Napoleon geheiratet zu haben und Penelope die, Odysseus als Gatten gehabt zu haben. / Die narrativen Welten, so hat man gesagt, seien immer kleine Welten, weil sie keinen maximalen und vollständigen Sachverhalt darstellen [...]. In diesem Sinn sind die narrativen Welten parasitär, denn wenn bei ihnen keine anderen Eigenschaften angegeben werden, nehmen wir an, sie hätten die Eigenschaften, die in der realen Welt gelten.“/ „Im allgemeinen [...] ist es so, daß, wenn wir uns auf fiktive Personen beziehen, wir dies aufgrund von Eigenschaften tun, die üblicherweise von den Enzyklopädien registriert werden [...].“ (Eco: Kant und das Schnabeltier, 373.)

14 „Es scheint [...], daß der Leser sehr viele Dinge über die reale Welt wissen muß, um sie als Hintergrund einer fiktiven Welt akzeptieren zu können. Und damit geraten wir in ein Dilemma: Einerseits kann ein fiktionales Universum, insofern es nur die Geschichte einiger Personen erzählt, die gewöhnlich an einem wohldefinierten Ort und in einer ebenso wohldefinierten Zeit spielt, als eine sehr kleine Welt erscheinen, unendlich viel kleiner als die reale Welt. Andererseits, insofern es die reale Welt als seinen Hintergrund in sich enthält und ihr nur ein paar Individuen, ein paar Eigenheiten und Ereignisse hinzufügt, ist es größer als die Welt unserer Erfahrung. In gewissem Sinne endet ein fiktives Universum nicht mit der Geschichte, die es erzählt, sondern dehnt sich ständig weiter aus. / In Wahrheit sind die fiktiven Welten zwar Parasiten der wirklichen Welt, aber die sind de facto ,kleine‘ Welten, die den größten Teil unserer Kenntnis der wirklichen Welt sozusagen ausklammern und uns erlauben, uns auf eine endliche und geschlossene Welt zu konzentrieren, die der unseren sehr ähnlich, aber ontologisch ärmer ist. Da wir ihre Grenzen nicht überschreiten können, fühlen wir uns gedrängt, sie in der Tiefe zu erforschen.“ (Eco: Im Wald der Fiktionen, 114-115.) 
In seinen Schriften setzt sich Eco immer wieder mit dem Thema „Interpretation“, mit historischen Praktiken des Interpretierens und mit tragfähigen Modellen von Interpretation auseinander. Auch und gerade seine Reflexionen über Fiktionales, dessen Konstitution und Diskursivierung ließen sich dem Interpretations-Thema subsumieren. Aber letztlich besteht auch auf dieser Ebene eher ein zirkuläres Verhältnis: Was als „Interpretation“ akzeptiert wird, resultiert aus „Faktizitäts“-Konzepten, und was als „faktisch“ akzeptiert wird, ist Ergebnis von „Interpretationen“. Das konkret-inhaltliche Interpretieren fiktionaler Welten steht dabei in einem Wechselbezug zum Wissen über reale Welten - respektive über das, was als real gilt.

Beim Eintritt in den Wald der Fiktionen wird von uns erwartet, daß wir den Fiktionspakt mit dem Autor unterschreiben und uns zum Beispiel darauf gefaßt machen, daß Wölfe sprechen können; wenn aber Rotkäppchen dann vom bösen Wolf gefressen wird, glauben wir, daß es tot ist (und dieser Glaube ist sehr wichtig für die Katharsis am Ende und für unsere große Freude über Rotkäppchens Auferstehung.) Wir glauben, daß der Wolf einen Pelz und aufrechtstehende Ohren hat [...]. Warum? Weil es in der Welt unserer Erfahrung so ist, also in jener Welt, die wir fürs erste [...] die reale oder wirkliche Welt nennen werden. ${ }^{15}$

Wer sich mit „Fiktionen“ befasst, befasst sich immer auch mit „Fakten“ und umgekehrt, selbst im Rahmen von als gültig angenommenen Differenzierungen zwischen dem einen und dem anderen. Über Fiktionen nachzudenken, kann insbesondere aufklärend wirken, gerade auch mit Blick auf den Umgang mit dem, was als ,faktisch', was als ,wirklich' und lebensrelevant verstanden wird. ${ }^{16}$ (Die Rekonstruktion ,vergangener' Wirklichkeit wird im Erfinden und Ausstatten fiktionaler Welten spielerisch eingeübt, schon deswegen greifen die kollektive Konstruktion der sogenannten wirklichen Welt respektive ihrer „Enzyklopädie“ und die Konstruktion fiktionaler Welten eng ineinander. ${ }^{17}$ ) Ein wichtiger Aspekt ergibt sich beim Blick auf fiktionale Welten in Relation $\mathrm{zu}$ als real geltenden auch aus

15 Eco: Im Wald der Fiktionen, 105-106.

16 „Das Nachdenken über die komplexen Beziehungen zwischen Leser und Geschichte, Fiktion und Realität, kann eine Form der Therapie sein gegen den Schlaf der Vernunft, der Ungeheuer gebiert. / In jedem Fall werden wir nicht darauf verzichten, literarische Fiktionen zu lesen, denn sie sind es, in denen wir nach einer Formel suchen, die unserem Leben einen Sinn gibt." (Eco: Im Wald der Fiktionen, 183.)

17 Die narrative Fiktion „bietet uns die Möglichkeit, unbegrenzt jene Fähigkeit auszuüben, die wir sowohl zur Wahrnehmung der Außenwelt wie zur Rekonstruktion der Vergangenheit brauchen. Die Fiktion hat die gleiche Funktion wie das Spiel. Spielend lernt das Kind zu leben, denn es simuliert Situationen, in denen es sich als Erwachsener befinden könnte. Und durch die narrative Fiktion üben wir Erwachsene unsere Fähigkeit, in die Erfahrung der Gegenwart wie der Vergangenheit eine Ordnung zu bringen.“ (Eco: Im Wald der Fiktionen, 174.) 
dem Umstand, dass erstere stets einer Intention, nämlich der ihrer Produzenten, entsprechen, während der Sinn des Faktischen keineswegs als gesichert gilt. Die Idee, Fakten als ,Botschaften` zu betrachten, ist dabei allzu verlockend, um nicht gelegentlich auch irreführenden Lese-Hypothesen $\mathrm{zu}$ erliegen. ${ }^{18}$

\section{Der Romanzo illustrato und die visuelle „Enzyklopädie“}

Auch wenn Ecos theoretische Erörterungen über Gegenstände und Praktiken der Interpretation, über Zeichen, ihre Vermittlungs- und Konstitutionsleistungen sowie über Narrationen zwischen Darstellung und Konstruktion sich zunächst primär auf Texte - im Sinne von: verbale schriftsprachliche Botschaften - beziehen, werden Bilder dabei doch vielfach einbezogen oder auf analoge Weise berücksichtigt.

In seinen Reflexionen über jene ,Enzyklopädien‘, welche das Weltverständnis historischer Kulturen prägen, vor allem auch deren Verständnis der eigenen Vergangenheit, nehmen Bilder einen zunehmend breiteren Raum ein.

Das bildliche Werk (der Kinofilm, die TV-Reportage, das Wandplakat, der Comic strip, das Foto) ist heute bereits ein integraler Bestandteil unseres Gedächtnisses. Was [...] eine fortgeschrittene Hypothese zu bestätigen scheint, nämlich daß die neuen Generationen sich, als Bestandteile ihres Verhaltens, eine Reihe von Bildern einverleibt haben, die durch den Filter der Massenmedien gegangen sind (und von denen einige aus den entlegensten Zonen der experimentellen Kunst unseres Jahrhunderts kommen). In Wahrheit braucht man nicht einmal von neuen Generationen zu sprechen: Es genügt, zur mittleren Generation zu gehören, um erfahren zu haben, wie sehr das gelebte Leben (Liebe, Angst oder Hoffnung) durch ,schon gesehene“ Bilder gefiltert wird. Überlassen wir es den Moralisten, diese Lebensweise ,aus zweiter Hand' (oder durch ,intermediäre Kommunikation') zu mißbilligen. Bedenken wir, dass die Menschheit nie anders gelebt hat, daß sie vor Nadar und den Gebrüdern Lu-

\footnotetext{
Soweit die ,positive' Botschaft. Die Kehrseite des Befundes ist aber ebenfalls zu berücksichtigen: „Doch wenn die erzählerische Aktivität so eng mit unserem Alltagsleben verbunden ist, könnte es dann nicht auch vorkommen, daß wir das Leben als Fiktion interpretieren und beim Interpretieren der Realität fiktive Elemente in sie einführen?“ (Eco: Im Wald der Fiktionen, 174.)

18 „Das Problem mit der wirklichen Welt ist, daß wir uns seit Jahrtausenden fragen, ob sie eine Botschaft enthält und ob diese Botschaft einen Sinn hat. Bei einer fiktiven Welt wissen wir mit Sicherheit, daß sie eine Botschaft darstellt und daß hinter ihr eine auktoriale Autorität steht, als ihr Schöpfer und als ein Ensemble von Instruktionen zu ihrer Lektüre. / So ist also unsere Suche nach dem Modell-Autor letztlich die Suche nach einem Ersatz für jenes andere Bild, das Bild eines Vaters, das sich im Nebel der Unendlichkeit verliert [...].“ (Eco: Im Wald der Fiktionen, 153.)
} 
mière nur andere Bilder benutzt hat, solche aus den Reliefs der heidnischen Tempel oder aus den Miniaturen der Apokalypse. ${ }^{19}$

Die insgesamt wachsende Aufmerksamkeit auf die Konstitution der ,Enzyklopädie‘ durch Bilderwelten nimmt auch auf die Gestalt von Ecos Büchern Einfluss. So etwa stellt sein katalogartiges Kompendium über Die Geschichte der legendären Länder und Städte die Gegenstände, um die es geht, nicht nur in Gestalt von Quellentextzitaten und sprachlichen Kommentaren dar, sondern auch in Form von Bildern; im Buch manifestieren sich also die zeitweilig zum „enzyklopädischen“ Wissen gehörenden Informationen in Text und Bild. ${ }^{20}$ Es geht mit den „terre e luoghi leggendari“ um Orte, die gleichsam im Schwellenbereich zwischen „Wirklichem“ und „Imaginärem“ bzw. „Fiktivem“ situiert sind - die also nicht (erkennbar) rein imaginär bzw. fiktiv sind, sondern zumindest zeitweilig für real gehalten und gesucht wurden - sowie um reale Orte, die man als Orte literarischer, mythischer oder legendenhafter Berichte betrachtet bzw. interpretiert hat. ${ }^{21}$

19 Eco, Umberto. Über Gott und die Welt. Essays und Glossen. Aus dem Italienischen von Burkhart Kroeber. München: dtv 1985 [1973], 214-215.

20 Vgl. Eco, Umberto. Die Geschichte der legendären Länder und Städte. Aus d. Ital. v. Martin Pfeiffer u. Barbara Schaden. München: Carl Hanser 2013. (It.: Storia delle terre e dei luoghi leggendari).

21 „Kataloge fiktiver Orte gibt es in großer Zahl (am vollständigsten ist das Manuale dei luoghi fantastici von Alberto Manguel und Gianni Guadalupi), aber wir behandeln hier keine ,erfundenen' Orte, denn sonst müssten wir auch das Haus von Madame Bovary, das Versteck Fagins in Oliver Twist oder die Festung Bastiani in Dino Buzzatis Tatarenwüste berücksichtigen. Unser Thema sind phantastische Orte, die bisweilen zum Ziel einer - kaum erfolgreichen - Suche fanatischer Leser werden. In anderen Fällen geht es um literarische Stätten mit realen Vorbildern: so suchen die Leser des Ulysses an jedem 16. Juni das Haus des Leopold Bloom in der Eccles Street in Dublin, sie besuchen den Martello Tower, der jetzt als Joyce-Museum dient [... . / Hier interessierten uns [...] Länder und Örtlichkeiten, an die sich heutzutage oder in der Vergangenheit Hirngespinste, Utopien und Illusionen geknüpft haben, weil viele Menschen wirklich glaubten, dass sie irgendwo existierten oder existiert hätten. / Allerdings sind dabei [...] Unterschiede zu berücksichtigen. Es hat Legenden über Länder gegeben, die es ganz sicher nicht mehr gibt, deren frühere Existenz sich aber nicht ausschließen lässt; so haben nach den letzten Spuren von Atlantis viele Menschen gesucht, [...]. Es gibt sagenhafte Länder, deren [...] Existenz zweifelhaft ist [...]; andere [...] sind mit Sicherheit Erfindungen eines Erzählers. Es gibt Länder wie das irdische Paradies oder das Land der Königin von Saba, deren Existenz nur in biblischen Quellen bezeugt ist, an die aber viele, auch Christoph Kolumbus, geglaubt haben, woraufhin sie dann auszogen, um reale Länder zu entdecken. Es gibt Länder, die wie das Reich des Priesters Johannes auf ein fingiertes Dokument zurückgehen und die gleichwohl Reisende dazu veranlasst haben, Asien und Afrika zu erkunden. Schließlich sind da auch heute noch existierende Örtlichkeiten, um die sich jedoch eine Mythologie rankt; hierher gehört Alamut, über dem der sagenhafte Schatten der Assassinen liegt, oder Glastonbury, das einst mit dem Gralsmythos verknüpft war [...]./ [...] [D]ie 
Schon in den Romanen Il nome della rosa und Il pendolo di Foucault haben die zunächst nur wenigen Bilder (es handelt sich um Karten und andere Funktionsgraphiken) keine rein ornamentale Funktion; sie dienen hier bereits einer Orientierung in den verbal dargestellten Welten der Romanfiktion, die an eine solch graphische Darstellung gebunden ist, tragen die Romanfiktion also mit. ${ }^{22}$ Il cimitero di Praga (dt. Der Friedhof in Prag) und La misteriosa fiamma della regina Loana dann enthalten umfangreiche Bildanteile, mit denen die jeweils dargestellte Romanwelt visuell inszeniert wird. Eco schließt hier an die Gattung des romanzo illustrato an, der - ähnlich wie der Historische Roman - im 19. Jahrhundert sein Profil annimmt. ${ }^{23}$ Eco betont explizit, welche Bedeutung die Bilder in seinen Romanen haben und wie stark er in seinen Vorstellungen davon, wie ein Roman auszusehen habe, als jugendlicher Leser durch Jules Verne und Emilio Salgari geprägt worden sei.

Weil die Bücher aus dem 19. Jahrhundert, die ich in meiner Kindheit gelesen hatte, alle illustriert waren, und weil sich so bei mir die Vorstellung herausbildete, ein richtiger Roman müsse bebildert sein. Zwar handelt es sich bei den in der Regina Loana zu findenden Bildern hauptsächlich um Zeitkolorit aus den 30er und 40er Jahren des 20. Jahrhunderts; aber inmitten dieser historischen Dokumente enthält dieser Roman auch einige rein imaginäre und aus der Welt der Literatur stammende Illustrationen, beispielsweise solche, die ursprünglich zu den Romanen von Jules Verne gehörten. Im Cimitero di Praga kommen nun

legendären Länder und Stätten sind von unterschiedlicher Art und haben nur eins gemeinsam: ob sie nun auf uralte Sagen zurückgehen [...] oder ob sie Produkte moderner Erfindung sind, sie haben Ströme von Glauben geschaffen. / Von der Wirklichkeit derartiger Illusionen handelt das Buch.“ (Eco, Umberto. Die Geschichte der legendären Länder und Städte. Aus dem Italienischen von Martin Pfeiffer und Barbara Schaden. München: Carl Hanser 2013, 7-9.)

22 Die mimetischen Bilder, die sein Imaginarium beeinflussten, hat Eco in die „Postille“ (Eco 1983) ausgelagert. Auch für Baudolino signifikant ist die enge Anknüpfung vieler beschreibender Textpassagen an bildliche Darstellungen aus dem Mittelalter, wie sie auch in Ecos genanntem Bildband zu sehen sind.

23 Romanzi illustrati sind seit Verne wichtige Schauplätze der Inszenierung von Faktischem und Fiktionalem. Jules Vernes Romane, die hier (neben Salgaris) den Prototypus bilden, sind einerseits klar Werke der Fiktion, nehmen andererseits aber auf vielfältiges Faktenwissen Bezug: auf naturwissenschaftliches, technisches, geo-, ethno- und historiographisches. Die Illustrationen der Romane Jules Vernes dienen einerseits der Illustration und damit der Bekräftigung der TextFiktionen, gleichsam der Effektverstärkung. Andererseits zeigen sie vielfach Realien, und zwar auf eine detaillierte und informative, ,allgemeinbildende“ Weise: Karten und Objektdarstellungen vermitteln Faktenwissen, auch an dieser Stelle. Gerade die Illustration im romanzo illustrato à la Jules Verne ist also eine Scharnierstelle, die fiktionale und faktuale Darstellungen miteinander verbindet, ja das eine ins andere umkippen lassen kann. Vgl. zu Vernes enzyklopädischen Ambitionen: Junkerjürgen, Ralf. Jules Verne. Darmstadt: wbg THEISS 2018, 8. 
tatsächlich fast alle Abbildungen aus dem Feuilletonroman des 19. Jahrhunderts vor, weil diese Gattung meine erste Leseerfahrung geprägt hat. ${ }^{24}$

Die Cimitero-Illustrationen sind als Bilder aus dem 19. Jahrhundert aber nicht allein dessen metonymische Repräsentationen, welche die Kohärenz der fiktionalen Romanwelt unterstützen sollen; sie gehören teilweise auch durch ihre Motive und ihren Erstpublikationskontext zu eben der Geschichte um eine angebliche Verschwörung, auf die der Roman Bezug nimmt.

\begin{abstract}
Was die Funktion dieser Bilder betrifft, so sollten diese einerseits die Atmosphäre jener Epoche ausstrahlen und so dem Cimitero in gewisser Hinsicht die narrative Aura des Ottocento verleihen. Andererseits habe ich zwischen diesen ursprünglich fiktionale Texte begleitenden Illustrationen auch einige Dokumente aus derselben Zeit versteckt, wie beispielsweise Titelseiten von La Libre Parole [eine antisemitische Zeitschrift]. Dies soll den Leser wachrütteln, er soll sich sagen: ,Also ist das nicht nur alles erfunden! Es ist wirklich passiert [...]. Die Abbildungen im Cimitero erfüllen also eine zweifache Funktion. ${ }^{25}$
\end{abstract}

Alle Cimitero-Kapitel sind bebildert; die Illustrationen korrespondieren jeweils zentralen Inhaltselementen der Kapitel und tragen in der Regel eine Bildlegende, die dem Erzähltext entnommen ist. Dabei sind die Illustrationen Bildzitate, die oft etwas ganz anderes illustriert haben. Manchmal ergeben sich auch Überschneidungen zwischen alter und neuer Bedeutung, etwa bei Darstellungen historischer Personen und Ereignisse.

24 Stauder, Thomas. Gespräche mit Umberto Eco aus drei Jahrzehnten. Berlin: LIT Verlag 2012, 267. Ausgangspunkt ist die Frage Stauders zur „Funktion der Illustrationen“ in Ecos Romanen und der Vergleich zwischen Königin Loana und Il cimitero di Praga: Unterscheidet sich nicht nur „die Art der Illustrationen in diesen beiden Romanen [...]“, sondern „auch deren jeweilige Rolle“? Eco weist darauf hin, dass auch die früheren Romane schon bebildert waren; er sei schon immer „ein multimedialer Erzähler“ gewesen. Dies begründet er mit seiner Leser-Sozialisation, also autobiografisch. (Stauder: Gespräche mit Umberto Eco aus drei Jahrzehnten, 266.)

25 Stauder: Gespräche mit Umberto Eco aus drei Jahrzehnten, 267. 


\section{Il cimitero di Praga - die im Spannungsfeld zwischen Faktualem und Fiktionalem konstruierte Geschichte einer Weltverschwörungs-Fiktion}

Il cimitero di Praga (2010) spielt in dem Jahrhundert, das den historischen und den illustrierten Roman hervorgebracht hat: im Ottocento. Der Roman nimmt insbesondere Bezug auf die vielleicht bekannteste und folgenreichste historische Weltverschwörungstheorie: auf die Geschichte der sogenannten Protokolle der Weisen von Zion. Eco hat sich mit diesen Protokollen, ihrer Rezeptions- und insbesondere ihrer Entstehungsgeschichte über längere Zeit befasst und selbst zur Frage der mutmaßlichen Entstehungsbedingungen geforscht. Das Thema, so Eco zwei Jahre nach Erscheinen des Romans in einem Interview, ${ }^{26}$ beschäftige ihn seit langem; gemeint ist das Thema Antisemitismus ${ }^{27}$ und - konkreter - die Geschichte eines mit antisemitischen Absichten gefälschten, angeblich, jüdischen' Dokuments: die sogenannten Protokolle der Weisen von Zion.

Die Protokolle der Weisen von Zion, übersetzt in verschiedenste Sprachen, existieren in zahlreichen Varianten; sie wurden seit ihrer Entstehung im späten 19. Jahrhundert viel rezipiert und oft ernst genommen; bis heute dienen sie antisemitischer Propaganda verschiedener Couleur. Es handelt sich in der Kernversion um das vorgebliche Protokoll eines Treffens von Rabbinern in Prag, die eine jüdische Weltverschwörung planen und dabei ausführlich erörtern, wie sie die Welt politisch und wirtschaftlich in ihre Gewalt bringen wollen (im Wesentlichen angelegt als Rede eines jüdischen Anführers vor den anwesenden „Weisen von Zion“). Inhaltlich zentrale Punkte des fingierten Dokuments sind u. a. die Idee des „Weltjudentums“, die Rechtfertigung auch unmoralischer und verbrecherischer politischer Mittel durch die Zwecke, die Kritik an Liberalismus und Demokratie als destabilisierend - wobei diese aber angeblich vom „Weltjudentum“ gefördert

26 Stauder: Gespräche mit Umberto Eco aus drei Jahrzehnten, 258-259, über den „Friedhof in Prag“: Frage T. S.: „Um die Genese Ihres neuen Romans nachzuzeichnen, müsste man auf Ihre Harvard Norton Lectures [= Im Wald der Fiktionen] vom Anfang der 90er Jahre verweisen, von denen eine auf die gefälschten Protokolle der Weisen von Zion eingeht, wobei Sie diese im Kontext anderer falscher Dokumente analysieren und auch deren Entstehungsgeschichte schildern.“

27 Eco hat 2002 in L'Espresso einen Artikel publiziert, der rhetorisch fragt, ob die Italiener Antisemiten sind („Gli italiani sono antisemiti?“, wieder abgedruckt in: Eco, Umberto. A passo di gambero, Guerre calde e populismo mediatico. Mailand: Bompiani 2006, 287-289): Hier weist er auf das Vorwort des Italieners Julius Evola für eine Auflage der Protokolle von 1937 hin. 
werden, weil sie die bestehenden Staaten schwächen. Zur Zerrüttung bestehender Systeme beitragen soll ferner die Verbreitung der Lehren von Marx, Nietzsche und Darwin durch Agenten des „Weltjudentums“. Weitere Mittel dieser angeblichen Verschwörergemeinschaft sind die Verursachung von Finanzkrisen, die Unterminierung der Macht der Kirche sowie Terroranschläge. Mit all dem wird vorgeblich ein Komplex von Zielen verfolgt: die Schwächung bestehender Staaten, bis die nervlich und finanziell zerrütteten Bürger um einen Herrscher bitten; vorbereitet wird ein Staatsstreich, der einen jüdischen König an die Macht bringt. Angestrebt ist die Installierung eines autokratischen Regimes mit scharfen Gesetzen und stark beschnittenen Bürgerfreiheiten. Zentral erscheint die Idee der Etablierung politischer Macht durch Terror, samt einem Hinweis auf die Möglichkeit, mittels der neuen Untergrundbahntunnel Städte in die Luft zu sprengen (die Metro in Paris befand sich ab 1897 in Konstruktion). Prägend für die sogenannten Protokolle ist die Integration diverser alter antisemitischer Stereotype (Rachsucht und Verschlagenheit der Juden, Geldgier und Neigung zu Verschwörungen) in eine moderne Machtfantasie politisch-ökonomischer Ausrichtung.

Eco interessiert sich vor allem für die Entstehungsgeschichte der Fälschung, für verwendete Textvorlagen und Ideenlieferanten der Protokolle; ${ }^{28}$ seine Überlegungen gelten dabei vor allem dem Anteil fiktionaler Texte an dem angeblich

28 Auf der Basis historischer Quellen und Hypothesen, darunter auch den Befunden Ecos, ergibt sich folgendes hypothetisch rekonstruierte Bild der Geschichte der Protokolle, die 1900 (vielleicht 1897/98) auf der Basis diverser Texte mit verwendeten motivlichen Anregungen verfasst werden (Indizien deuten auf die Pariser Bibliothèque nationale de France als Arbeitsplatz). Pjotr Ratschkowski, 1885-1902, Leiter des russischen Geheimdienstes in Paris (Ochrana), lässt der Hypothese nach einen antiliberalen satirischen Text des französischen Schriftstellers Maurice Joly umschreiben (oder schreibt ihn selbst um); so entsteht ein antisemitisches Pseudo-Protokoll. Das Motiv ist mutmaßlich der Versuch, den russischen Zaren gegen liberalistische Kräfte aufzuhetzen. Einer anderen Hypothese zufolge ist Urheberin oder Veranlasserin die russische Okkultistin Juliana Glinka. Unklar bleibt, ob es ein französisches Original gab (wie oft behauptet wird). Die erste Publikation der Protokolle erfolgt in Russland 1903; besonders wirkmächtig wird eine weitere russische Publikation 1905 in einer Schrift von Sergej Nilus. Nilus verbreitet das Gerücht, Theodor Herzl, Begründer der Zionistischen Bewegung, sei der Urheber. Es entstehen Übersetzungen in verschiedene Sprachen, zwischen 40 und 80 Seiten lang; 1911 wird eine deutsche Fassung durch den Antisemiten Ludwig Müller von Hausen („Gottfried zur Beek“) verbreitet. 1921 erfolgt eine Verknüpfung der (angeblichen) jüdischen Weltverschwörung mit der angeblichen Weltverschwörung der Illuminaten durch Nesta Websters Schrift World Revolution. The Plot against Civilization. Verschiedene spekulative Theorien über die Illuminaten als die eigentlichen Drahtzieher schließen sich an. Im selben Jahr kommt es zur Entlarvung der Protokolle als Fälschung (durch einen Artikel von Philip Graves in der Londoner Times von 1921). 1933-1934 findet ein Prozess um die Authentizität der Protokolle vor dem Obergericht des Kantons Bern (Schweiz) statt; laut Gerichtsbeschluss kann die Echtheit des Textes nicht erwiesen werden; die Verurteilung befürwortender Artikel als „Hetzliteratur“ wird gerechtfertigt. Trotz der Aufklärung ab 1921 erfolgen 
auf faktische Ereignisse bezogenen Dokument. Neben anderen Schriften wird der Cimitero-Roman zum Medium der Rekonstruktion. Auf dieser Basis ergeben sich folgende Dependenzen: Hauptvorlage der Protokolle bildet Maurice Jolys Dialogue aux enfers entre Machiavel et Montesquieu, 1864 anonym publiziert in Brüssel, ohne inhaltliche Bezugnahme auf Juden. Jolys Satire gilt der autoritären Politik Napoleons III., dessen politische Skrupellosigkeit ,Machiavelli‘ vertritt. 40 \% der Protokolle sind wörtliche Übernahmen aus Jolys Text. Jolys Vorlage wiederum waren französische Unterhaltungsromane des 19. Jahrhunderts von Eugène Sue (Mystères du peuple; Le Juif errant) und Alexandre Dumas d. Ä. (Joseph Balsamo, hier wird eine Weltverschwörung der Jesuiten dargestellt). Eco selbst ist maßgeblicher Entdecker dieser Abhängigkeiten zwischen Romanliteratur und Protokollen. Eine weitere Vorlage der Protokolle (neben Jolys Text) ist der Roman Biarritz (1868) von Hermann Goedsche (Pseudonym: „Sir John Retcliffe“), genauer: die Darstellung eines geheimen Treffens 1860 auf dem Prager Friedhof; der Fiktion nach erfolgen regelmäßige Treffen von Vertretern des Judentums zwecks Besprechung eines Welteroberungsplans. Den Roman kennzeichnen antisemitische Klischees und eine antiliberale Grundtendenz in Anknüpfung an ältere Vorstellungen über eine jüdische Weltverschwörung. Goedsches Roman von 1868 wurde 1876 als Tatsachenbericht publiziert; Auszüge erschienen als „Rede des Rabbiners“ an verschiedenen Stellen (in antiliberalen, katholischen Presseorganen), teils auch als Zusammenfassungen der 12 „Reden“. Angebliche Quelle ist der Bericht eines englischen Diplomaten „Sir John Readcliff“.

Il cimitero di Praga erzählt eine fingierte Entstehungsgeschichte der Protokolle, in welche aber die von Historikern (und Eco selbst) recherchierten Bedingungszusammenhänge (Vorgeschichte, Vorlagen, mutmaßliche Verfasser, Auftraggeber, Drahtzieher) in Form einer kohärenten Geschichte integriert sind. ${ }^{29}$ Die in die Handlung einfließenden Informationen zur Vorgeschichte der fraglichen Verschwörung sind teilweise Resultate von Ecos eigenen textkritischen Forschungen und wollen als Beiträge zur Analyse eines historischen Falles ernstgenommen werden. Die Romanfiktion soll hier durchaus der Vermittlung von historisch-faktualem Sachwissen dienen. Die paratextuellen Elemente, die dem Romantext beigefügt sind, übernehmen daher eine wichtige Orientierungsfunktion.

weiterhin Publikationen (in verschiedenen Sprachen); die Protokolle dienen zu politischen Propagandazwecken u. a. durch Henry Ford, durch die deutschen Nationalsozialisten, durch Antisemiten verschiedener Länder - und dies bis heute (vor allem in Russland und in Ländern der islamischen Welt).

29 Hier besteht eine Parallele zu Baudolino, wo es um die erfundene Entstehungsgeschichte des (tatsächlich existierenden!) Briefs des „Priesters Johannes“ geht. 
Will Eisner hat die Geschichte der Protokolle in der Graphic Novel The Plot (2005) nacherzählt. Für diese hat Eco ein Vorwort verfasst. Wie es dort heißt, muss die Geschichte der Protokolle erzählt werden, um „die große Lüge und den Hass, den sie hervorbringt“, zu bekämpfen. ${ }^{30}$ Thomas Stauder fragt Eco, ob er dies als „Plädoyer für das politische Engagement der Literatur“ verstehe und die „soziale[... V Verantwortung von Romanautoren“ betonen wolle. ${ }^{31}$ Eco bestätigt das, weitet den Appell aber über den Roman hinaus auf Essays und andere publizistische Formen aus.

Was Il cimitero di Praga betrifft, so muss ich [...] sagen, dass es zwar selten ist, Romane hauptsächlich aus propagandistischen Zwecken zu verfassen, dass ich diesmal aber dieser Zielsetzung sehr nahe kam. Denn ich hatte festgestellt, dass nicht nur ich selbst, sondern auch viele Andere [...] auf die Fälschung der Protokolle in gelehrten Aufsätzen und Büchern hingewiesen hatten, dass dies aber die öffentliche Meinung kaum beeinflusste und die in den Protokollen enthaltene Idee von der jüdischen Weltverschwörung weiter zirkulierte. Deshalb hatte ich die Hoffnung, durch die Schilderung der Entstehungsgeschichte dieser Fälschung in der unterhaltsamen Form eines Romans vielleicht ein größeres Publikum zu erreichen. Da die Übersetzungsrechte des Cimitero - soweit ich gehört habe - auch von einem arabischen Verleger erworben wurden, kann mein Roman nun möglicherweise auch im arabischen Sprachraum dazu beitragen, die Lügen der Protokolle entlarven zu helfen. ${ }^{32}$

Inhalte, Konstruktion und Schreibweise der Romanerzählung sind auf die Auseinandersetzung mit dem Problemkomplex um Fälschung, Lüge und (Selbst-)Täuschungen abgestimmt. Ecos fiktiver Verfasser der Protokolle ist der 1830 in Turin geborene, nun in Paris lebende Jurist Simon Simonini, ein Fälscher, der durch seine Fälschungsaktivitäten in diverse politische Ereignisse verwickelt wird und diese auch aktiv und gezielt beeinflusst. Aus seiner Perspektive wird der größte Teil der Handlung erzählt: Simonini verfasst Tagebuchaufzeichnungen auf den Rat eines Nervenarztes namens „Doktor Froïde“ hin, den er konsultiert, weil er unter einer Amnesie leidet: Er hat die meisten Erinnerungen an sein Leben verloren. Durch den Schreibakt soll Simonini versuchen, sie wiederzugewinnen. Bei

30 Eco, Umberto. Einführung zu Will Eisner: Das Komplott. München: DVA-Verlag 2007, unpag. Orig.: Secret Story of The Protocols of the Elders of Zion. New York: W. W. Norton 2005. Vgl. zum Thema auch Stauder: Gespräche mit Umberto Eco aus drei Jahrzehnten, 259.

31 Stauder: Gespräche mit Umberto Eco aus drei Jahrzehnten, 259-260.

32 Stauder: Gespräche mit Umberto Eco aus drei Jahrzehnten, 260-261. Stauder weist darauf hin, dass die Behauptungen der Protokolle in den arabischen Ländern aktuell noch von vielen ernst genommen werden; Eco ergänzt, auch in Deutschland würden die Protokolle weiterhin gelesen, obwohl ihre Veröffentlichung verboten sei; der Text sei im Internet aber verfügbar. In Italien sei das Buch in okkultistisch-esoterischen Buchhandlungen greifbar. (Vgl. Stauder: Gespräche mit Umberto Eco aus drei Jahrzehnten, 261.) 
diesem Versuch schaltet sich - wie es scheint - ein in der Nachbarwohnung lebender Abbé namens Dalla Piccola ständig ein, indem er die Aufzeichnungen Simoninis parallel zu ihrer Entstehung kommentiert und um eigene Texte erweitert. Allerdings trifft Simonini mit dem Abbé nie zusammen und erwägt schon bald, dieser könne ein abgespaltener Teil seiner selbst sein, was sich bestätigt. Simonini hofft, seine mentale Krise überwunden zu haben und nicht verrückt zu sein. Allerdings wird diese Einschätzung vom Romantext selbst unterlaufen. Simoninis Pariser Leben ist vor allem geprägt durch Kontakte zu Geheimdiensten (zu piemontesischen, französischen und deutschen), zu Geheimbündlern, Geheimgesellschaften und Verschwörern. In diesem Kontext verfasst er die (sogenannten) Protokolle der Weisen von Zion, nutzt (,stiehlt') Vorlagen und Anregungen, wird aber auch selbst von einem Plagiator bestohlen. Der Romanschluss suggeriert, dass er als Metro-Bombenattentäter zu Tode kommt. Die Persönlichkeitsspaltung Simoninis wird von Eco psychologisch entsprechend geläufigen Interpretationsmustern motiviert, desgleichen seine vielfachen Ressentiments, seine stereotypen Denkweisen, der Hass auf Juden, Jesuiten, Freimaurer, Franzosen, Deutsche, Italiener und Frauen. Während in Baudolino das Fälschen als ein Spiel erscheint, das „Geschichte macht“, dabei aber niemandem wesentlich schadet (im Gegenteil soll die Fälschung des Priester-Johannes-Briefs einmal dabei helfen, Friedrich Barbarossa von seinem gewalttätigen Kreuzzug abzulenken), wird hier die Gegengeschichte erzählt: über Fälschungen als Instrumente des Hasses und der Aufhetzung, verfasst nicht von einem lebensfrohen Lügengeschichtenerfinder, sondern von einem psychisch gestörten, ja wahnsinnigen Menschenhasser.

Die Konstruktion der Erzählung wie auch der Inhalt (vor allem die Lebensgeschichte eines Fälschers) bedingen es, dass die Äußerungen der Romanfiguren weitenteils als Gerüchte, unbegründete Meinungen, Vorurteile, bloße Behauptungen, Irrtümer oder Lügen erscheinen.

Dabei gehen Wahres und Erlogenes, Wirkliches und Fingiertes bis zur Ununterscheidbarkeit ineinander über - und das genau entspricht auch der Praxis des Fälschers, um die es inhaltlich geht. Der Roman ist hinsichtlich seiner stofflichen Dimension ähnlich doppelgesichtig: Er bezieht sich auf eine erhebliche Zahl historischer Prozesse, Ereignisse und Figuren. ${ }^{33}$ Andere Figuren sind fiktiv, darunter

33 Im Roman auftretende historische Figuren sind u. a. Giuseppe Garibaldi, Ippolito Nievo, „Doktor Froïde“, ein Nervenarzt in Paris, Dr. Charcot, Abbé Barruel, Pjotr Iwanowitsch Ratschkowski, von 1884-1902 als russischer Geheimagent in Paris tätig (möglicherweise beteiligt an der Entstehung der Protokolle der Weisen von Zion), Alexandre Dumas d. Ä., Hermann Goedsche sowie andere Romanciers und Publizisten, die durch Publikationen zur Geschichte der Protokolle beigetragen haben. 
Simon Simonini, dessen Namen Eco allerdings der Schrift eines Verschwörungstheoretikers, des Abbé Barruel, entnahm. ${ }^{34}$

\section{Das Spiel der Bilder}

Die Bilder im Cimitero sind in einem mehrfachen Sinn als ,dokufiktional' beschreibbar: Sie sind erkennbar Zitate historischer Bildmaterialien, die in ihren ursprünglichen historischen Präsentationskontexten zu erheblichen Teilen die Funktion hatten, etwas $\mathrm{zu}$,dokumentieren', und zwar auf eine Weise, die sich aus rückblickender Perspektive und von einem aufgeklärten Standpunkt her als ,fiktional' beschreiben lässt: Mit diversen Bild-,Dokumenten' geht es um die ,zionistische Weltverschwörung' aus der Perspektive derjenigen, die diese Bilder seinerzeit veröffentlichten (teils auch schon aus derjenigen der Bild-Produzenten). Die Bilder hatten den Status von ,Dokufiktionen“ in dem Sinn, dass sie mittels bildgenerierender Konstruktion von Figuren und Szenen etwas ,dokumentieren“ sollten, was sich anders nicht ,dokumentieren' ließ - und dies auf eine durchaus geläufige Weise der Wissensvermittlung und öffentlichen Information, nämlich im Medium der Druckgraphik. ,Doku'-Fiktionen bietet Ecos romanzo illustrato aber auch in dem Sinn, als er im Rahmen einer Roman-Fiktion historische Dokumente präsentiert, die mittelbar Einsichten in reale historische Zusammenhänge katalysieren - insbesondere Einsichten in die Funktionsmechanismen einer historischen Bildkultur und in deren politische Dimension.

Von der einen Seite betrachtet, zeigen sich dem Romanleser solche Bilder, die zumindest teilweise als Pseudo-Plausibilisierungen der jüdischen Weltverschwörung fungierten und insofern ,lügenhafte‘ Bilder sind, die den falschen

34 Barruel, 1741-1820, war Jesuit und verfasste eine Geschichte des Klerus während der französischen Revolution, in der er u. a. behauptete, fanatische Jakobiner hätten 1792 katholische Priester gebraten und gegessen. Warnende Verschwörungstheorien, die sich an die Idee der Existenz einer Geheimgesellschaft knüpften, entstehen zur Zeit der französischen Revolution. 17971798 verfasst der Abbé Barruel seine Mémoires pour servir à l'histoire du jacobinisme - wie Eco sagt, „als Antwort auf die Französische Revolution“: „ein dem Anschein nach historisches Werk, das sich jedoch wie ein Schauerroman liest.” (Eco: Im Wald der Fiktionen, 176.) Geheimagenten verfassen für Napoleon einen Bericht, der auf die Angaben des Marquis de Luchet und des Abbé Barruel zurückgreift. 1806 bekommt Barruel einen Brief des Hauptmanns Simonini, der die Juden ins Spiel bringt: „[...] die Freimaurerei seien [sic!] von Juden gegründet worden, und sämtliche existierenden Geheimgesellschaften seien von Juden infiltriert” (so Simoninis Behauptung). „Es scheint, daß der Brief von Simonini in Wirklichkeit vom Agenten des Polizeiministers Fouché stammte, der sich Sorgen über Napoleons Kontakte zu den französischen Juden machte.“ (Eco: Im Wald der Fiktionen, 177.) 

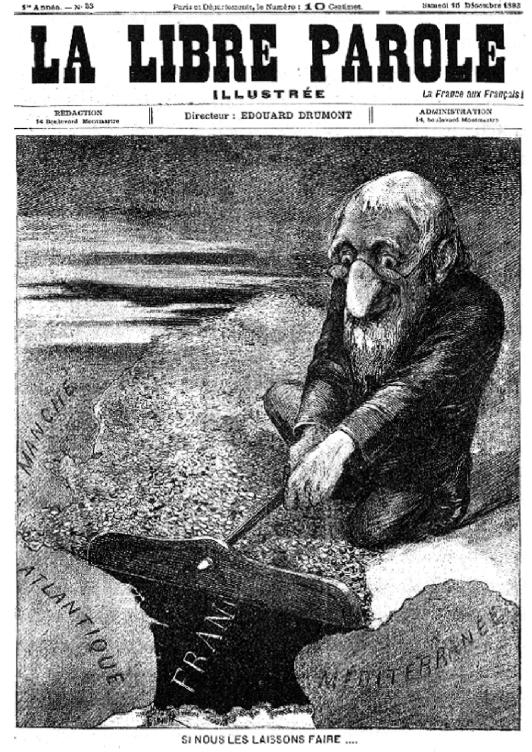

...in den folgenden Jahren ließ Simonini sich oft bei ihm blicken, erst in der Ligue Antisémite, die Drumont gegründet hatte, und dann in der Redaktion seiner Zeitung La Libre Parole... (S. 402)
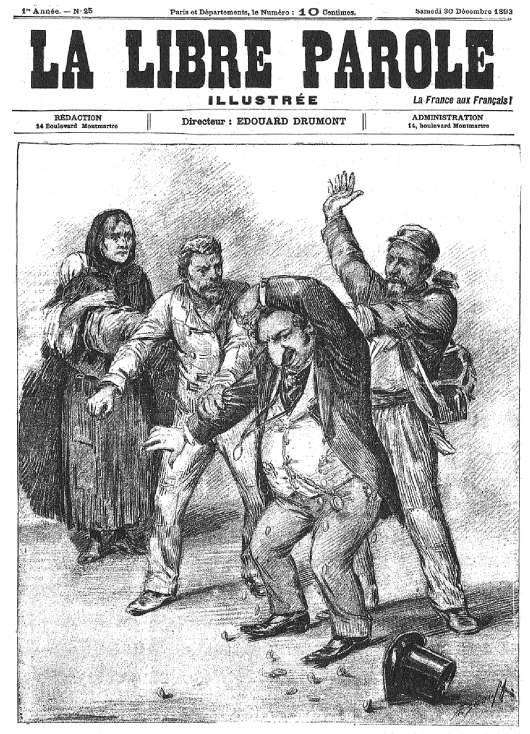

...und jetzt beherrschen sie die Börsen und sind die Herren des Kreditwesens. Deshalb kann der Sozialismus nicht umhin, antisemitisch zu sein... (S.393)
Abb. 1: Die in einem nationalistischen Blatt publizierte Karikatur eines raffgierigen Juden illustriert exemplarisch die Beteiligung des Journalismus an der Verbreitung antisemitischer Stereotype - und damit eine publizistische Praxis der Bekräftigung fiktionaler Konstrukte wie das der jüdischen Weltverschwörung. Zum typischen Bildercode des Antisemitismus gehören physiognomische Merkmale des Geld-,Raffers'. Als authentische Titelseite einer Zeitung ist das zu Ecos Sammlung historischer Bildmaterialien gehörige Blatt allerdings ein ,echtes‘ Dokument, so ,falsch' seine Suggestionen auch sind. (aus: Eco: Der Friedhof in Prag, 403.)

Abb. 2: Eine weitere antisemitische Karikatur stellt die aus Sicht des Blattes legitime Reaktion der ,guten Franzosen' auf das Judentum dar, das hier wiederum stereotyp ins Bild gesetzt wird. Die agitatorisch-performative Dimension dieser authentischen, dabei in den Kontext eines propagandistischen Fiktionszusammenhangs gehörenden und insofern lügenhaften Illustration wird durch das Sujet besonders betont. (aus: Eco: Der Friedhof in Prag, 394.) 
Anschein faktualer Berichterstattung über die Verschwörer von Prag bekräftigen sollten. Von der anderen Seite betrachtet, sind die historischen Bildbestände faktuales Material, an dem sich ablesen lässt, welche historischen Faktoren in der Geschichte dieser Verschwörungstheorie ineinandergegriffen haben. Der Roman selbst ,dokumentiert‘ also mit fiktionalen Mitteln, wie Verschwörungstheoretiker mit fiktionalen Mitteln zu ,dokumentieren' pflegten. Aber er setzt dabei auf die Differenz zwischen rationalen und irrationalen Interpretationspraktiken und verortet seine impliziten Leser auf der Seite der rationalen Interpreten, die (unter anderem) mit der Differenzierung zwischen Faktischem und Fiktionalem arbeiten, auch wenn, ja gerade weil sie um deren historische Bedingtheit und Relativität wissen.

Die Bilder illustrieren den Text nicht einfach nur, sie treiben ein eigenes Spiel. Mit dem Romantext sind sie dadurch verbunden, dass ihre Bildlegenden Zitate aus dem Romantext bilden, allerdings aus dem Zusammenhang herausgelöst. Dadurch ,sprechen' die Bilder nochmals nach, was im Roman seitens einer der Erzählerinstanzen gesagt wird - wie es in Romanen des 19. Jahrhunderts üblich ist.

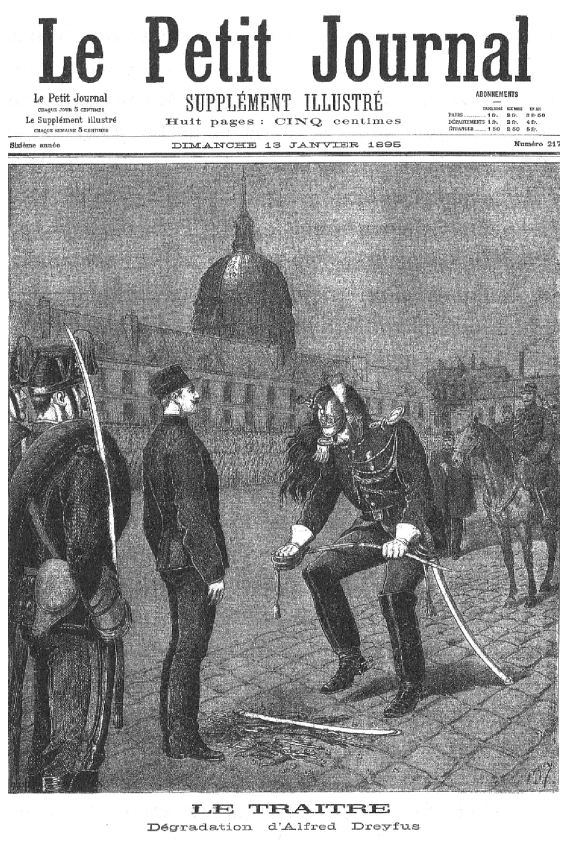

...ein riesiger Gardefeldwebel mit Federbuschhelm tritt vor den Hauptmann, reißt ihm die Epauletten, die Tressen, die Knöpfe vom Rock, nimmt ihm den Säbel ab, zerbricht ihn auf seinem Knie und wirft die beiden Hälften dem Verräter vor die Füße... (S. 431)
Abb. 3: Die Dreyfus-Affäre bildete einen Kulminationspunkt antisemitischer Tendenzen in Frankreich zur Zeit der Romanhandlung. Die Zeitungs-Titelillustration zeigt die Degradierung des Leutnants Dreyfus, nicht in karikaturistischem, sondern in realistischem Stil: eine Szene, die so oder ähnlich stattgefunden hat. Nicht die karikaturistische Überzeichnung des Juden Dreyfus und nicht die Szene selbst lassen das Bild zum Funktionsteil eines Gewebes aus Fiktionen werden, sondern der mittelbare Bezug zur öffentlichen Stimmungslage, die die Dreyfus-Affäre auslöste. (aus: Eco: Der Friedhof in Prag, 433.) 
Eine ganze Reihe der in den Roman integrierten Bilder präsentieren sich schon insofern als performanzaffin, als sie Schauplätze zeigen, mehrfach verbunden mit Performanzen, politischen, kämpferischen, auch okkultistisch-spiritistischen.

\section{Fazit}

Il cimitero di Praga, so lässt sich bilanzieren, fügt sich in einen ganzen Komplex von Texten Ecos über Verschwörungstheorien und konkreter über die Protokolle ein. Hierbei spielen Bilder als literarische Gestaltungselemente sowie zumindest implizit als Teil des thematisierten Gegenstandes (der Verschwörungstheorie, des politischen Propagandaapparates, der historisch wirkmächtigen „Lügen“) eine signifikante Rolle. Indem er teilweise historische Bildmaterialien eindeutig ideologischen Charakters reinszeniert, macht Eco deutlich, dass und wie auch Bilder zur Genese und Popularisierung von Fiktionen beitragen, die als solche nicht durchschaut werden sollen, ja an deren Wahrheit womöglich die Bildproduzenten selbst glauben. Außerdem demonstriert er dadurch, dass er Propaganda-Bilder mit anderen, ideologisch ,neutraleren` Bildern mischt (etwa mit Porträts historischer Personen), wie sich Fiktionales und Faktendarstellung bis zur Ununterscheidbarkeit vermischen können. Dabei finden sich auf manchen Bildern selbst bereits faktuale und fiktionale Bildelemente kombiniert; so mischen sich ,Dämonen' unter Menschen etc. Dass wiederverwendete Bilder von Figuren aus Romanfiktionen dazu dienen, vorgeblich ,historische' Personen zu porträtieren, vertieft die Konfusion ebenfalls.

Wie es der Text-Kritik bedarf, um einen Text wie die Protokolle als das zu durchschauen, was sie sind, so bedarf es auch der Bild-Kritik. Hier setzt Ecos Roman vor allem auf für die Leser erkennbare Brüche und Diskrepanzen. Dies gilt insbesondere für die „Diana“-Sequenz. Die im Roman auftretende Figur wird, so die Suggestion, gleich mehrmals „porträtiert“; tatsächlich handelt es sich um Bilder verschiedener Provenienz, und die Figuren ähneln einander nicht einmal. Der vergleichende Blick wird zum entlarvenden Blick. Als ein wichtiger Bestandteil kritischer Analyse von Texten und Bildern erscheint - wie schon in Il nome della rosa - der genaue Blick; der Detektiv muss nicht nur die Codes kennen, nach denen man Texte entschlüsselt, er sollte auch eine Brille nutzen.

Eco weiß um die Macht der Interpretationen und erörtert immer wieder (in theoretischen Schriften wie in Romanen) deren Bedingtheiten - die jeweilige „Enzyklopädie“ des Interpreten, die angewandten Codes, die interpretationslenkenden Ausgangshypothesen etc. - also: die Relativität von Interpretationen. Relativ in diesem Sinn der durch diverse Faktoren bedingten Kontextabhängigkeit sind 
Text-Interpretationen ebenso wie Interpretationen von Geschichte und historischen Zusammenhängen, von kulturellen Tatsachen, ja selbst die von Natur, insofern diese stets aus dem Blickwinkel historisch-kultureller Codes und „Enzyklopädien“ wahrgenommen wird. Bei aller Betonung dieser Bedingtheit von Interpretation hat Eco - im Gegenzug zu dem, was er an der Dekonstruktion wahrnahm und kritisierte - nie die Differenzierung zwischen angemessenem und unangemessenem Interpretieren preisgegeben, auch wenn, wie er weiß, diese Differenzierung wiederum notgedrungen ein Akt der Interpretation ist. Nicht preisgegeben wird auch die Differenzierung zwischen Faktualem und Fiktionalem; Eco spricht von der wirklichen und den möglichen Welten, wobei er allerdings deutlich macht, dass es sich vor allem um eine heuristisch notwendige Differenzierung handelt und man es im konkreten Fall einer Entscheidung über Faktizität und Fiktionalität eben wiederum mit Interpretationen zu tun hat.

Dass Eco an der Differenzierung zwischen angemessenen und unangemessenen Interpretationen festhält, bietet die Grundlage seiner Kritik an politischen Lügen, die auf unangemessenen Interpretationen beruhen, sei es, dass reale Interpretanda absichtsvoll verzerrt interpretiert werden, sei es auch, dass sie erfunden werden. Eine solche auf Täuschung respektive auf Propaganda abzielende Interpretation ebnet die Differenz zwischen dem Faktischen und dem Fiktionalen ein, ohne den Interpretationscharakter der eigenen Darstellung zu erkennen zu geben. Oft beginnen die Propagandisten und Tatsachenfälscher dann ja sogar, an ihre eigenen Lügen zu glauben. Dies allerdings ist alles andere als eine beruhigende Vorstellung.

\section{Literatur}

Cohn, Norman. Warrant for Genocide: The Myth of the Jewish World Conspiracy and the Protocols of the Elders of Zion. New York: Harper \& Row 1966.

Eco, Umberto. Il nome della rosa. Milano: Bompiani 1980.

Eco, Umberto. Der Name der Rose. Aus dem Italienischen von Burkhart Kroeber. München: Carl Hanser 1982 [1980].

Eco, Umberto. Postille a ,ll nome della rosa: Milano: Bompiani 1983.

Eco, Umberto. Semiotik und Philosophie der Sprache. Aus dem Italienischen von Christiane Trabant-Rommel und Jürgen Trabant. München: Fink 1985 [1984].

Eco, Umberto. Über Gott und die Welt. Essays und Glossen. Aus dem Italienischen von Burkhart Kroeber. München: dtv 1985 [1973].

Eco, Umberto. Semiotik. Entwurf einer Theorie der Zeichen. Aus dem Italienischen von Günter Memmert. München: Fink 1987 [1976].

Eco, Umberto. Lector in fabula. Die Mitarbeit der Interpretation in erzählenden Texten. Aus dem Italienischen von Heinz-Georg Held. München: Carl Hanser 1987 [1977]. 
Eco, Umberto. Il pendolo di Foucault. Milano: Bompiani 1988.

Eco, Umberto. Die Grenzen der Interpretation. Aus dem Italienischen von Günter Memmert. München: dtv 1999 [1990].

Eco, Umberto. Baudolino. Milano: Bompiani 2000.

Eco, Umberto. Kant und das Schnabeltier. Aus dem Italienischen von Frank Herrmann. München: Carl Hanser 2003.

Eco, Umberto. „Über einige Funktionen der Literatur“. In: Umberto Eco. Die Bücher und das Paradies. München: Carl Hanser 2003 [2002], 9-24.

Eco, Umberto. Im Wald der Fiktionen. Sechs Streifzüge durch die Literatur. Aus dem Italienischen von Burkhart Kroeber. München: dtv 2004 [1994].

Eco, Umberto. La misteriosa fiamma della regina Loana. Milano: Bompiani 2004.

Eco, Umberto. Einführung zu Will Eisner: Das Komplott. München: DVA-Verlag 2007, unpaginiert. Orig.: Secret Story of The Protocols of the Elders of Zion. New York: W. W. Norton 2005.

Eco, Umberto. „Gli italiani sono antisemiti?“ In: Umberto Eco. A passo di gambero, Guerre calde e populismo mediatico. Milano: Bompiani 2006, 287-289.

Eco, Umberto. Il cimitero di Praga. Milano: Bompiani 2010.

Eco, Umberto. Der Friedhof in Prag. Aus dem Italienischen von Burkhart Kroeber. München: Carl Hanser 2011.

Eco, Umberto. Die Geschichte der legendären Länder und Städte. Aus dem Italienischen von Martin Pfeiffer und Barbara Schaden. München: Carl Hanser 2013.

Eco, Umberto. Numero zero. Milano: Bompiani 2015.

Joly, Maurice. Dialogue aux enfers entre Machiavel et Montesquieu, ou La politique de Machiavel au XIXe siècle par un contemporain. Brüssel: Mertens 1864.

Junkerjürgen, Ralf. Jules Verne. Darmstadt: wbg THEISS 2018.

Mersch, Dieter. Umberto Eco. Zur Einführung. Hamburg: Junius 1993.

Retcliffe, John [= Hermann Goedsche]. Biarritz. Berlin : Liebrecht 1868.

Rollin, Henri. L'apocalypse de notre temps. Les dessous de la propagande allemande d'après des documents inédits. Paris: Gallimard 1939.

Searle, John. „The Logical Status of Fictional Discourse“. In: New Literary History 6 (1975), 319-332.

Stauder, Thomas. Gespräche mit Umberto Eco aus drei Jahrzehnten. Berlin: LIT Verlag 2012.

Wilson, N. L. „Linguistic Butter and Philosophical Parships“. In: Journal of Philosophy 64 (1967), 55-67. 